\title{
Correction to: Meeting report from the fourth meeting of the Computational Modeling in Biology Network (COMBINE)
}

Dagmar Waltemath ${ }^{1 *}$, Frank T. Bergmann ${ }^{2}$, Claudine Chaouiya ${ }^{4}$, Tobias Czauderna ${ }^{3}$, Padraig Gleeson ${ }^{5}$, Carole Goble ${ }^{6}$, Martin Golebiewski ${ }^{7}$, Michael Hucka ${ }^{2}$, Nick Juty ${ }^{8}$, Olga Krebs ${ }^{7}$, Nicolas Le Novère ${ }^{8,14}$, Huaiyu Mi ${ }^{9}$, Ion I. Moraru ${ }^{10}$, Chris J. Myers ${ }^{11}$, David Nickerson ${ }^{12}$, Brett G. Olivier ${ }^{13}$, Nicolas Rodriguez ${ }^{14}$, Falk Schreiber ${ }^{3,15,16}$, Lucian Smith², Fengkai Zhang ${ }^{17}$ and Eric Bonnet ${ }^{18,19,20^{*}}$

\section{Correction}

Following publication of the original article [1], the author requested this addition to the 'Acknowledgements' section of the article: "DW is funded by the German Federal Ministry of Education and Research (e: Bio program SEMS, FKZ 031 6194)".

\section{Author details}

'Department of Systems Biology and Bioinformatics, University of Rostock, Rostock, Germany. ${ }^{2}$ Department of Computing and Mathematical Sciences, California Institute of Technology, Pasadena, CA, USA. ${ }^{3}$ P PK Gatersleben, Gatersleben, Germany. ${ }^{4}$ Instituto Gulbenkian de Ciência - IGC, Rua da Quinta Grande, Oeiras, Portugal. ${ }^{5}$ Department of Neuroscience, Physiology and Pharmacology, University College London, London, UK. ${ }^{6}$ School of Computer Science, The University of Manchester, Manchester, UK. ${ }^{7}$ Heidelberg Institute for Theoretical Studies, Heidelberg, Germany. ${ }^{8}$ EMBL-European Bioinformatics Institute, Wellcome Trust Genome Campus, Hinxton, Cambridge, UK. ${ }^{9}$ Department of preventive medicine, Keck School of Medicine, University of Southern California, Los Angeles, CA, USA. ${ }^{10}$ Center for Cell Analysis and Modeling, University of Connecticut Health Center, Farmington, CT, USA. ${ }^{11}$ Department of Electrical and Computer Engineering, University of Utah, Salt Lake City, USA. ${ }^{12}$ Auckland Bioengineering Institute, University of Auckland, Auckland, New Zealand. ${ }^{13}$ Systems Bioinformatics, VU University Amsterdam, Amsterdam, The Netherlands. ${ }^{14}$ The Babraham Institute, Babraham Research Campus, Cambridge, UK. ${ }^{15}$ Martin Luther University Halle-Wittenberg, Halle, Germany. ${ }^{16}$ Monash University, Melbourne, Australia.

${ }^{17}$ Computational Biology Unit, Laboratory of Systems Biology, National Institute of Allergy and Infectious Diseases, $\mathrm{NIH}$, Bethesda, MD, USA. ${ }^{18}$ Institut Curie, Paris, France. ${ }^{19}$ INSERM U900, 75248 Paris, France. ${ }^{20}$ Mines ParisTech,

77300 Fontainebleau, Paris, France.

Published online: 09 August 2018

\section{Reference}

1. Waltemath $D$, et al. Meeting report from the fourth meeting of the

Computational Modeling in Biology Network (COMBINE). Stand Genomic Sci. 2014;9(3):1285. https://doi.org/10.4056/sigs.5279417.

\footnotetext{
* Correspondence: dagmar.waltemath@uni-rostock.de; eric.bonnet@curie.fr 'Department of Systems Biology and Bioinformatics, University of Rostock, Rostock, Germany

${ }^{18}$ Institut Curie, Paris, France
} 